\title{
Prenatal ultrasonography screening fetal autosomal trisomy
}

\author{
PAN Yu-ping ${ }^{1,2, a}$ \\ ${ }^{1}$ Medical College, Eastern Liaoning University, Dandong, Liaoning118003 China \\ ${ }^{2}$ Department of Ultrasound, Shengjing Hospital of China Medical University, \\ Shenyang ,Liaoning110004 China] \\ aemail :Panxy900@sina.com
}

Keywords: Amniocentesis; Cordocentesis ; Karyotyping ;Ultrasonography; Trisomy.

Abstract: Objective To investigate the value of prenatal ultrasonography screening fetal autosomal trisomy(trisomy 21, trisomy 18, trisomy 13).Methods Amniocentesis and cordocentesis were performed on 6425 pregnant women respectively with indications for prenatal diagnosis to detect karyotype of the fetus during second trimester and late pregnancy, The detection rate of trisomy 21 , trisomy 18 , trisomy 13 was compared in pregnant women of different indications, To analyze the relationship between the ultrasonography abnormalities and these trisomy. Results In chromosomal karyotypes analysis of 6425 pregnant women by Amniocentesis and cordocentesis, 98 trisomy (66 trisomy 21, 28 trisomy 18, 4 trisomy 13) were detected, The detection rate of trisomy 1.53\%; There were 424 in 6425 pregnant women with ultrasonography abnormalities, 30 trisomy (16 trisomy 21, 12 trisomy 18,2 trisomy 13 ) were found within them and the detection rate of trisomy was $7.08 \%$, the detection rate of trisomy detected by ultrasound $(7.08 \%)$ was higher than the 18-trisomy high-risk group $(1.42 \%)$, advanced age group $(0.88 \%)$, the Down's syndrome high risk group $(0.83 \%)$ $(0.00 \%)$ (all $P<0.05)$; no significant difference was detected in trisomy detection rate between the abnormal ultrasound group (7.08\%) and the NTD high risk group $(4.76 \%)(P>0.05)$.Conclusions Prenatal ultrasonography is great value in screening fetal autosomal trisomy.

\section{Introduction}

Genetic ultrasonogram can markedly improve the detection rate of trisomy ${ }^{[1]}$. In this study, we performed amniotic fluid cell analysis and fetal cord blood karyotyping in 6425 pregnant women, in order to explore the clinical value of ultrasound screening on fetal autosomal trisomy during second trimester and late pregnancy.

\section{Clinical data and Methods}

Clinical data all subjects are pregnant women sought medical examination or consultation in Shengjing Hospital of China Medical University. Six thousand four hundred sixty six patients with indications of amniocentesis and fetal cord blood puncture were included from July 5, 2009December 31, 2012, aged 18 48 years, with a mean age of $(30.6 \pm 4.5)$ years old and $17 \sim 37$ weeks of gestational age. All indications of amniotic fluid puncture and umbilical cord blood sampling were detected during prenatal diagnosis, including: advanced maternal age (35 years old and above), high risk of Down syndrome (rate $\geq 1 / 270$ ), abnormal ultrasound findings, high risk of neural tube defects (NTD) and 18-trisomy syndrome, abnormal pregnancy history, history of chromosomal abnormalities of the parents, family history of genetic diseases, maternal mental retardation, drug history, viral infection and other history of obvious exposure to teratogenic factors.

All pregnant women signed informed consent for post-puncture karyotyping analysis.

\section{Methods}

Sample collection and cell culture: ultrasound-guided amniocentesis was performed and 20 30 $\mathrm{ml}$ amniotic fluid was drawn for laboratory culture. 
Umbilical cord puncture was also performed under guidance of ultrasound, and $2 \mathrm{ml}$ blood sample was placed in anticoagulant sodium heparin-treated disposable collection tube for laboratory culture.

Chromosome karyotype analysis: after G-band staining, cultured cells were observed and 15 30 metaphase images were counted. Three to five karyotypes were analyzed and abnormal karyotype was carefully observed and analyzed.

I have obtained permission from my institution's ethics committee to perform this study and approval from my institutions review board or ethics committee.

Instruments and ultrasound methods: Voluson E8 ultrasound diagnostic apparatus by GE company was used, with probe frequency of $4 \sim 6 \mathrm{MHz}$. Through abdominal multiplanar scanning, fetal head, face, neck, chest and abdomen, internal organs, limbs and spine etc. were scanned and suspicious sites were carefully observed. During follow-up, routine measurement of relevant data was performed including fetal biparietal diameter, limb length, amniotic fluid volume and placental thickness etc. Results were recorded.

Statistical analysis: SPSS17.0 software was used for statistical analysis. Count data of each group were compared using $\chi^{2}$ test and $P<0.05$ was considered statistically significant.

\section{Results}

Cell culture: 6466 pregnant women received amniocentesis, and amniotic fluid cell culture was succeeded in 6425 patients, with a success rate of $99.37 \%$.

Chromosome karyotyping of pregnant women: In chromosomal karyotypes analysis of 6425 pregnant women by amniocentesis and cordocentesis, 98 trisomy were detected with a detection rate of $1.53 \%$. There were 424 in 6425 pregnant women with ultrasonography abnormalities, and 30 of them was found with trisomy with a detection rate of $7.08 \%$.

The detection rate of trisomy in 6425 pregnant women with amniotic fluid and umbilical cord blood puncture indications was shown in Table 1.

Table 1 The detection rate of trisomy in 6425 pregnant women with amniotic fluid and umbilical cord blood puncture indications

\begin{tabular}{lllll}
\hline Indications & $\begin{array}{l}\text { Patients } \\
\text { number }\end{array}$ & $\begin{array}{l}\text { Normal } \\
\text { karyotype }(\mathrm{n})\end{array}$ & Trisomy $(\mathrm{n})$ & $\begin{array}{l}\text { Detection rate } \\
(\%)\end{array}$ \\
\hline Abnormal ultrasound & 424 & 394 & 30 & 7.08 \\
High risk of NTD & 21 & 20 & 1 & 4.76 \\
High risk of 18-trisomy & 211 & 208 & 3 & 1.42 \\
Advanced maternal age & 1137 & 1127 & 10 & 0.88 \\
High risk of Down syndrome & 2770 & 2747 & 23 & 0.83 \\
$\begin{array}{l}\text { Other puncture indications } \\
\text { Combined with other puncture }\end{array}$ & 789 & 789 & 0 & 0 \\
indications & 1073 & 1042 & 31 & 2.89 \\
Total & 6425 & 6327 & 98 & 1.53 \\
\hline
\end{tabular}

$\chi^{2}$ test found that the detection rates of chromosomal abnormalities among groups were significantly different $\left(\chi^{2}=104.236\right.$ and $\left.P<0.001\right)$. Inter-group comparison by $\chi^{2}$ test found that trisomy detection rate of the abnormal ultrasound group (7.08\%) was significantly higher than that of the 18-trisomy high-risk group $(1.42 \%)\left(\chi^{2}=9.141, P=0.003\right)$, the advanced maternal age group $(0.88 \%)\left(\chi^{2}=47.485\right.$, $P=0.000)$ and the Down syndrome high risk group $(0.83 \%)\left(\chi^{2}=87.886, P=0.000\right)$. However, no 
significant difference was detected in trisomy detection rate between the abnormal ultrasound group $(7.08 \%)$ and the NTD high risk group $(4.76 \%)\left(\chi^{2}=0.165, P=0.684\right)$.

Correlation of trisomy with abnormal ultrasound findings: in this study, abnormal ultrasound findings of trisomy included NF thickening, short FL and HL(Figure 1), polyhydramnios(Figure 2), choroid plexus cyst, mild dilatation of lateral ventricle, hand and foot abnormalities (Figure 3), heart malformations (mainly ventricular septal defect) (Figure 4), cisterna magna broadening (Figure 5), omphalocele, .permanent right umbilical vein (Figure 6), pulmonary cystadenoma, facial abnormalities such as micrognathia and microtia, low-set ears, lip and palate cleft, mild renal pelvis dilatation, intrauterine growth retardation (IUGR), pleural effusion, skin edema, single umbilical artery, abnormal diastolic flow of umbilical artery, umbilical cord cyst, esophageal atresia, duodenal stenosis or atresia, bacterial intestinal dilatation and holoprosencephaly and so on.

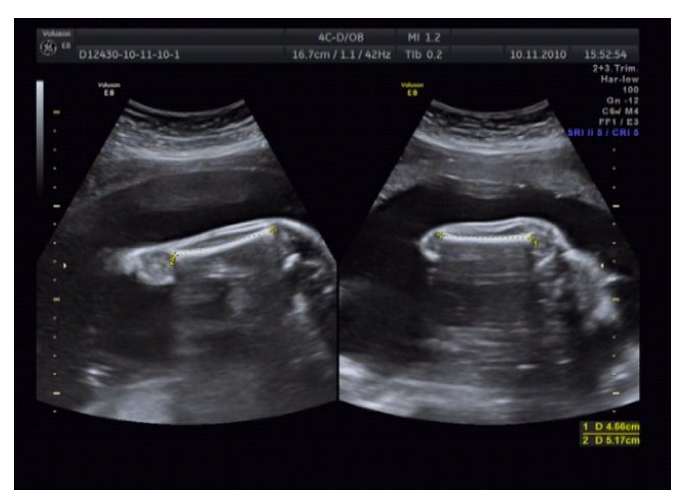

Figure 1. short FL and HL

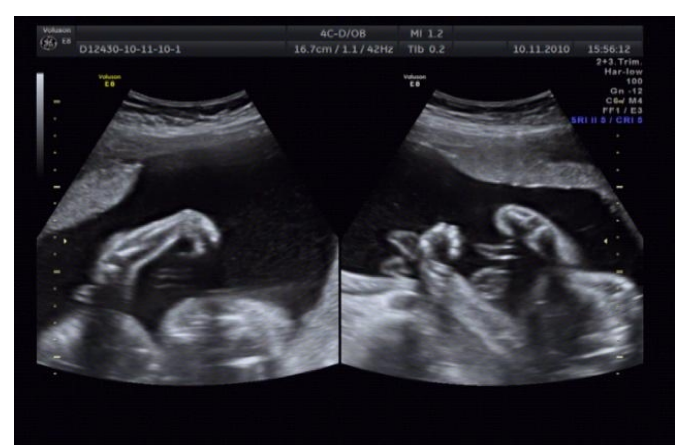

Figure 3. Clenched hand Figure

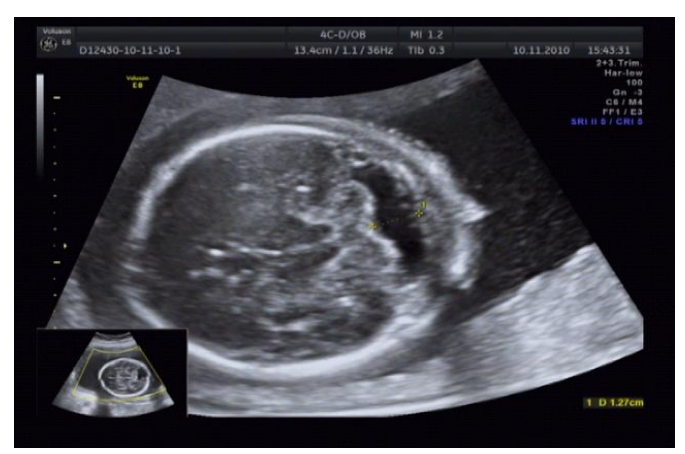

Figure 5. cisterna magna broadening

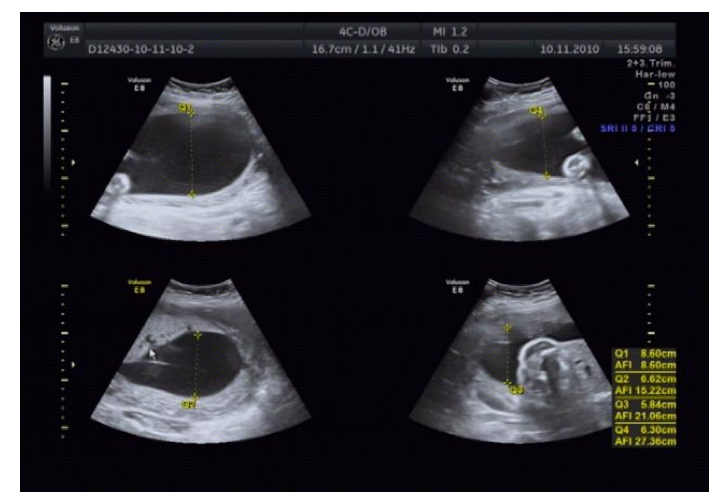

Figure 2. polyhydramnios

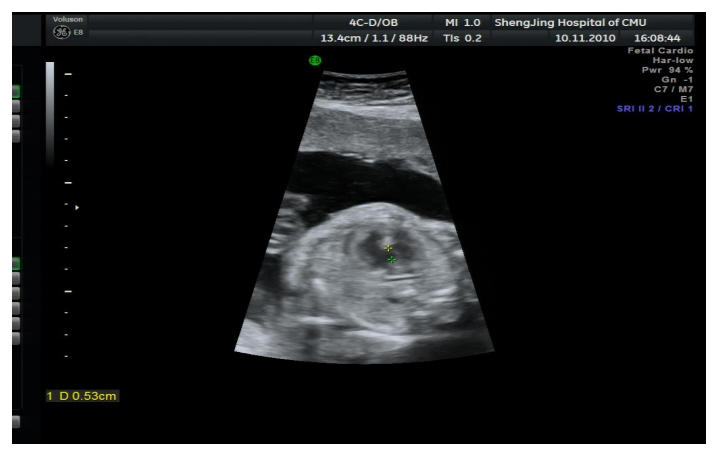

Figure 4. Ventricular septal defect

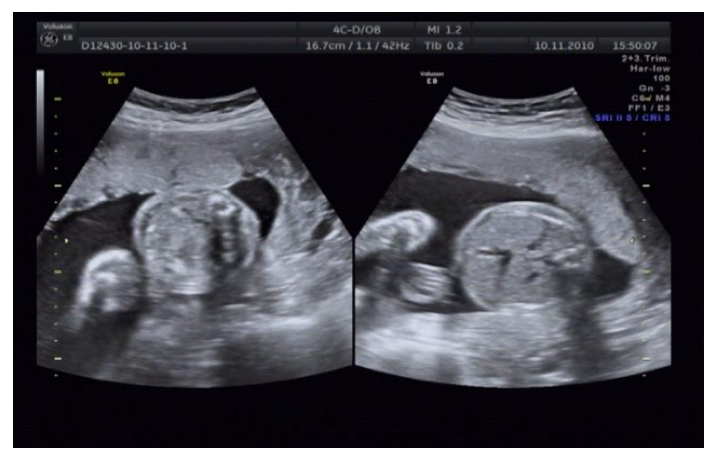

Figure 6. permanent right umbilical vein

Figure 1 - Figure 6 showed symptoms of the same pregnant woman, who was 24 years old and 30 weeks of gestation age, BPD $74.4 \mathrm{~mm}$, FL51.7 mm, HL45.6 mm, including short FL (Figure 1)(AFL/EFL $=51.7 / 57.86=0.89)$, short HL( Figure 1$)(\mathrm{AHL} / \mathrm{EHL}=45.6 / 55.24=0.83)$, polyhydramnios (amniotic fluid index $27.36 \mathrm{~cm}$ )(Figure 2), clenched hand (Figure 3), ventricular septal defect5.3 mm,(Figure 4), cisterna magna broadening $12.7 \mathrm{~mm}$ (Figure 5), abdominal wall echo interrupt, 
interrupt diameter $22 \mathrm{~mm}$, omphalocele20.5 $\mathrm{mm} \times 20.5 \mathrm{~mm}$ and .permanent right umbilical vein (Figure 6), Ultrasonic examination showed multiple malformations of the fetus. Karyotyping of umbilical cord blood was $47, \mathrm{XN},+18$.

\section{Discussion}

Common methods for prenatal diagnosis of trisomy include serum screening, ultrasonography and karyotype analysis, the first two as non-invasive examinations and the last one as invasive examination, which is considered the gold standard for prenatal diagnosis of trisomy. Invasive prenatal diagnostic techniques have certain risks. Since most of trisomy fetuses are delivered by reproductive age women ${ }^{[2-3]}$, non-invasive screening methods have grown in popularity. Serum screening has certain shortcomings, while fetal trisomy is an important genetic basis for congenital malformations ${ }^{[4]}$, so the value of ultrasonography screening for trisomy has been emphasized greatly.

Value of ultrasound screening for fetal autosomal trisomy ultrasound screening of fetal autosomal trisomy includes two aspects, the first being anatomical abnormalities of the fetus, i.e., the more deformities identified, the greater risk of trisomy. Most of the trisomy fetuses have 2 or more abnormal ultrasound findings, so detection of more than 2 ultrasonic signs suggests possible aneuploidy ${ }^{[5]}$. In this study, multiple fetal malformations were identified in 26 trisomy,. The second aspect of ultrasound screening for trisomy is markers for chromosomal abnormalities, which indicate increased risk of chromosomal diseases among fetuses and are referred as the "ultrasound soft markers." Some soft markers are closely correlated with detection of trisomy ${ }^{[7]}$.

The detection rate of trisomy in pregnant women with abnormal ultrasound findings was significantly higher than that of advanced maternal age group, Down syndrome high-risk group or 18-trisomy high-risk group. Thus, abnormal ultrasound findings are very important for the detection of trisomy.

In this study, ultrasonographic findings of trisomy were similar to these reported in previous literatures ${ }^{[8-9]}$. Specifically, previous study suggested that a NF thickness $\geq 6 \mathrm{~mm}$ indicate a 17 times higher risk for fetal 21-trisomy ${ }^{[6]}$, and we identified 8 cases of 21-trisomy with increased NF thickness in our study. In addition, it has been reported that polyhydramnios was associated with increased risk of fetal chromosomal abnormalities, while 12 pregnant women with trisomy fetuses in our study had polyhydramnios, among which 7 cases were 21 -trisomy and 5 cases were 18-trisomy. In this study, the most common heart defects associated with trisomy were ventricular septal defects, accounting for $66.67 \%$, which were closely correlated with chromosomal abnormalities, consistent with literature report ${ }^{[10]}$. Some study reported that 21 - and 18-trisomy have distinct cardiac abnormalities ${ }^{[11]}$. Other reports suggested that $16 \%$ of fetuses with abnormal cardiac structure alone had chromosome abnormalities, and $66 \%$ fetuses combined with other structural abnormalities had chromosomal abnormalities. In this study, 9 cases of ventricular septal defects were detected among trisomy fetuses ( 1 case of simple ventricular septal defect and 8 cases of ventricular septal defect combined with multiple fetal malformations).

False-negative rate of ultrasound screening as shown in Table 1, ultrasound screening alone detected 30 cases of trisomy fetuses, while ultrasound screening combined with puncture indications detected 20 cases of trisomy, with a total trisomy detection rate of 51.02\%(50/98). This suggests that ultrasound is an effective screening method for trisomy. However, 48 fetuses with normal ultrasound evidences were confirmed as trisomy through other further puncture detections, accounting for $48.98 \%(48 / 98)$ of the trisomy detection rate, suggesting that the normal ultrasound results in these patients are false negative. If we have not performed karyotype analysis based on maternal age, serum screening and other adverse reproductive history of the pregnant women, we would have missed the diagnosis of trisomy in these patients. Therefore, joint screening methods can reduce the false negative rate of ultrasound screening and improve the detection rate of trisomy. 


\section{Conclusion}

In summary, ultrasonography is of great value in screening fetal autosomal trisomy during second trimester and late pregnancy.

\section{Acknowledgement}

The subject is funded by the research foundation of Eastern Liaoning University, project number $2015 \mathrm{JC} 014$.

\section{REFERENCES}

[1] Fang YM, Benn P, Egan JF. Use of the genetic sonogram in the United States in 2001 and 2007[J]. J Ultrasound Med, 2008, 27 (11): 1543-1548.

[2] Czuba B, Borowski D, Cnota W, et al. Ultrasonographic assessment of fetal nuchal translucency (NT) at 11th and 14th week of gestation-Polish multicentre study [J] . Neuro Endocrinol Lett, 2007, 28 (2): $175-181$.

[3] Sághy T. Periodontal changes in Down syndrome. A literature review [J] . Fogorv Sz, 2008, 101 (3): $113-118$.

[4] Franssen MT, Korevaar JC, Tjoa WM, et al. Inherited unbalanced structural chromosome abnormalities at prenatal chromosome analysis are rarely ascertained through recurrent miscarriage $[\mathrm{J}]$. Prenat Diagn, 2008, 28 (5): 408-411.

[5] Raniga S,Desai Parikh H. Ultrasonographic soft markers of aneuploidy in second trimester are we lost . Medscape genetal Medcine,2006,8 (1): 9.

[6] Vanden H of MC, Wilson RD. Fetal soft markers in Obstetric ultrasound . J Obstet Gynaecol Can, 2005,27(6):592-636.

[7] Michiel C.Van den H of MD, Halifax NS R.Douglas Wilson, MD,Philadelphia PA etal.Fetal soft markers in obstetric ultrasound.J Obstet Gynaecol Can, 2005, 27 (6): 592 .

[8] Falcon O,Auer M. Screening for trisomy 21 by fetal tricuspid regurgitation, nuchal translucency and maternal serum free beta-hCG and PAPP-A at $11+0$ to $13+6$ weeks . Ultrasound Obstet Gynecol,2006,27(2):151-155.

[9] Cicero S. Maternal serum biochemistry at 11-13(+6) weeks in relation to the presence or absence of the fetal nasal bone on ultrasonography in chromosomally abnormal fetuses: an updated analysis of integrated ultrasound and biochemical screening. Prenat Diagn, 2005,25(11): 977-983.

[10] Meberg A, Hals J, Thaulow E. Congenital heart defects chromosomal anomalies, syndromes and extracardiac malformations $[\mathrm{J}]$. Acta Paediatr, 2007, 96 (8): 1142-1145.

[11] Hajdú J, Beke A, Pete B, et al. Prenatal diagnosis of the atrioventricular septal defect and its effect on the outcome of the pregnancies. Orv Hetil, 2005, 146 (34):1775-1780. 